\title{
Médiévales
}

Langues, Textes, Histoire

51 | automne 2006

L'Occident sur ses marges ( $\mathrm{VI} \mathrm{e}^{\mathrm{e}} \mathrm{X} \mathrm{I}^{\mathrm{e}}$ siècles)

\section{La fission du noyau : anciens et nouveaux centres dans l'espace alpin (fin $\mathrm{VII}^{\mathrm{e}}$-début $\mathrm{X}^{\mathrm{e}}$ siècles)}

The Nuclear Fission: Ancient and New Centers in the Alpine Region. from the End of the 7th Century to the Beginning of the 10th Century.

\section{Rodolphe Dreillard}

\section{CpenEdition}

Journals

Édition électronique

URL : https://journals.openedition.org/medievales/1540

DOI : $10.4000 /$ medievales. 1540

ISSN : 1777-5892

Éditeur

Presses universitaires de Vincennes

Édition imprimée

Date de publication : 1 décembre 2006

Pagination : 53-68

ISBN : 978-2-84292-193-4

ISSN : 0751-2708

Référence électronique

Rodolphe Dreillard, "La fission du noyau : anciens et nouveaux centres dans l'espace alpin (fin viledébut $x^{e}$ siècles) », Médiévales [En ligne], 51 | automne 2006, mis en ligne le 27 mars 2009, consulté le 23 avril 2022. URL : http://journals.openedition.org/medievales/1540 ; DOI : https://doi.org/10.4000/ medievales. 1540

Ce document a été généré automatiquement le 23 avril 2022

Tous droits réservés 


\title{
La fission du noyau : anciens et nouveaux centres dans l'espace alpin (fin $\mathrm{VII}^{\mathrm{e}}$-début $\mathrm{X}^{\mathrm{e}}$ siècles)
}

\author{
The Nuclear Fission: Ancient and New Centers in the Alpine Region. from the \\ End of the 7th Century to the Beginning of the 10th Century.
}

Rodolphe Dreillard

1 La question de la structuration progressive des royaumes issus du traité de Verdun (843) a fait l'objet d'études riches, que ce soit au sujet des rapports des rois avec les aristocraties ${ }^{1}$, de la réorganisation, autour de chaque souverain, d'une structure palatiale et d'une chancellerie ${ }^{2}$, du contrôle des réseaux épiscopaux et monastiques ${ }^{3}$. Le rôle qu'ont joué les relations avec les marges dans la construction des nouveaux centres de pouvoir constitue également une piste à explorer. Un tel projet doit s'appuyer sur les outils d'étude des marges définis par les géographes et les spécialistes des études politiques, et surtout sur le couple centre et périphérie qui permet de mettre en valeur la domination plus ou moins importante qu'exerce une zone géographique sur une autre. Par centre, on entendra le "point à partir duquel se distribue les phénomènes dans l'espace ${ }^{4} "$, soit une acception à la fois géographique, c'est-à-dire l'espace qui concentre les lieux, les individus, les institutions contribuant à affirmer une domination sur son environnement, et socio-politique, à savoir l'ensemble de ces instances de domination. La périphérie se définit donc d'abord comme ce qui n'est pas le centre, dans une relation dynamique entre espace dominant et espace dominé ${ }^{5}$. Là encore, elle peut prendre un sens strictement géographique, étant alors strictement synonyme de marge, d'espace bordier, limitrophe, mais également social et politique, désignant tout ce qui est placé dans la dépendance des instances centrales. Dans cette acception, il est donc possible que, paradoxalement, une zone centrale géographiquement parlant, comme le sont les Alpes en Europe, puisse se trouver en position de périphérie politique, sociale ou économique. Dans un souci de clarté, on parlera donc du Centre politique et du centre géographique ${ }^{6}$. 
2 Dans la période qui va de la conclusion du traité de Verdun à la stabilisation de nouvelles entités politiques dans la première décennie $d u \mathrm{x}^{\mathrm{e}}$ siècle, l'échec de l'ensemble lotharingien transforme l'ancienne région centre du pouvoir carolingien, entre Meuse et Rhin, en zone de marche disputée entre les royaumes occidentaux et orientaux, mais aussi, dans sa partie sud, entre souverains cis- et transalpins. L'arc alpin forme donc une frontière entre l'Italie et le reste de l'ancien empire, au moment où, paradoxalement, Rome développe une nouvelle centralité, qui rayonne au-delà de l'ensemble franc.

3 Les relations entre ces différentes puissances, royaumes franc occidental, germanique et italien, et Rome, sont donc dépendantes de cette périphérie lotharingienne et alpine. Une part de leur attitude à l'égard des autres fut déterminée par ce rapport à l'ancien Centre marginalisé et par leurs liens avec les centres de pouvoir qui apparaissent alors dans cette périphérie. Un point non négligeable de cette évolution est en outre la façon dont, dans les années 880 , les entités situées à la périphéries de l'ancien royaume des Francs unitaire sont les premières à s'autonomiser, voire à se détacher durablement du pouvoir royal carolingien, pour constituer des principautés (Aquitaine, Bavière) ou des royaumes frontaliers (Bourgogne), dont l'évolution est en grande partie commandée par leur aptitude, ou non, à réactiver des réseaux anciens, à les développer et à les utiliser pour générer de nouvelles légitimités ou tirer partie de leur position d'interface. Cette analyse des réseaux se fonde essentiellement sur les apports de la sociologie relationnelle telle qu'elle a été développée par R. S. Burt ${ }^{7}$ et des disciples, et en particulier sur la théorie des "trous structuraux », points d'interruption en marge de deux réseaux dans lesquels peuvent venir se glisser de nouveaux Centres en position d'interface, fondés sur un rôle de contrôle d'accès (gatekeeper) qui relie entre eux deux réseaux distincts (network switch). Cette théorie purement socio-économique trouve néanmoins une traduction spatiale dans la mesure où elle peut être liée à la notion géographique d'interface, c'est-à-dire de "ligne de contact entre deux systèmes ou ensembles distincts ${ }^{8}$. Il a été remarqué que ces zones de limites sont des espaces dynamiques d'échange, d'innovation de modification d'une partie par l'autre. Elles sont donc propices à générer des centres autonomes distincts de ceux, lointains, qui voient leur domination parvenir à son ultime limite au contact d'un autre pouvoir.

Dans les quelques pages qui suivent, c'est à partir de l'exemple de la zone alpine que je veux développer cette intuition, en revenant tout d'abord sur le statut qui a été accordé aux Alpes à travers l'histoire des partages du royaume des Francs carolingien, puis en étudiant sa position géographique et politique spécifique et les entités qui la structure, enfin, en étudiant quelles en furent les conséquences dans les quatre dernières décennies $\mathrm{du} \mathrm{IX}^{\mathrm{e}}$ siècle, quand les aristocraties localement implantées y contestèrent la domination carolingienne.

L'atome et ses électrons : le rapport centre-périphérie

dans l'empire de Charlemagne et de Louis le Pieux

vu au prisme des projets de partage

5 Le centre de l'arc alpin central, du Montcenis au Col da Fuorn (Offenpass) constitue un espace particulier dans le monde franc, longtemps marginalisé par sa position sur les limites sud du royaume ${ }^{9}$, loin des centres du pouvoir neustriens et austrasiens dont il est coupé par le plissement jurassien ${ }^{10}$. La conquête du royaume lombard en 774 ne modifie pas fondamentalement la donne, mais aboutit plutôt à un paradoxe : placée au 
centre géographique de l'ensemble carolingien, cette zone n'en reste pas moins une périphérie.

6 La maîtrise de cet espace par les Francs est passée par la prise de contrôle de trois grands points de passage associés à trois centres locaux qui les contrôlent et tiennent les routes conduisant sur le versant sud. Ces «micro frontières" sont autant d'électrons gravitant autour du noyau carolingien, que les sources baptisent « cluses ». Une lettre d'Alcuin à l'évêque Remi de Coire atteste du rôle qu'elles jouent dans la structuration des échanges entre les centres du nord et ceux du sud du massif montagneux ${ }^{11}$. Il s'agit des deux monastères de Saint-Maurice d'Agaune et de Novalèse ${ }^{12}$ et du siège épiscopal de Coire. Les deux premiers tiennent respectivement les cols du Grand Saint-Bernard et du Montcenis ainsi que les cités italiennes de Suse et d'Aoste, elles aussi sous pouvoir Franc ${ }^{13}$. Le troisième contrôlait les passes grisonnes, voie la plus rapide pour gagner l'Italie depuis la Germanie ${ }^{14}$. Quel est le destin de ces espaces dans le monde carolingien?

7 Les zones constituant le centre des royaumes des deux cadets de Charles dans son projet de partage de $806{ }^{15}$, et de Louis le Pieux, dans l'ordinatio de $817{ }^{16}$, sont des zones périphériques : l'Aquitaine et l'Italie en 806 , l'Aquitaine et la Bavière en 817 , ce qui marque une rupture par rapport à l'ancienne pratique, encore utilisée en 769 , qui cherchait à partager équitablement le cœur de la Francia entre les héritiers. Dans ces projets de partage, Charles et Lothaire sont les seuls à conserver le plein contrôle du centre, les périphéries étant appelées à former des entités plus ou moins autonomes selon le cas, et donc à structurer leur propre centralité.

8 Le partage de 806 ne fait qu'entériner la situation existant depuis les années 780 , où les royaumes d'Aquitaine et d'Italie ont d'une certaine façon prolongé leur existence autour de leurs centres historiques, Toulouse et Pavie, devenus résidences du sous-roi carolingien. Le partage confirme la façon dont certaines périphéries géographiques structurent leur propre centralité politique. Les trois royaumes partagent une périphérie commune : les Alpes, auxquelles les trois rois ont accès par le biais des trois espaces définis ci-dessus : le Montcenis à Louis, le Saint-Bernard à Charles et la Rhétie à Pépin ${ }^{17}$.

9 En 817, le rattachement de Rome, et donc du regnum italiae, au centre, modifie la donne sans la modifier fondamentalement. La primauté de Lothaire se traduit par la transmission du titre impérial, mais aussi, spatialement, par la constitution d'un « hyper centre » réunissant les deux capitales, Rome et Aix, qui a pour conséquence le passage de l'ensemble de l'arc alpin central, et de tous les grands cols, essentiels à la continuité de son pouvoir, sous sa domination. En revanche, le choix de l'Aquitaine et de la Bavière pour l'établissement de Pépin et Louis correspond là encore à la reconnaissance de deux périphéries aptes à générer leur propre centralité. La différence fondamentale résidait dans le fait que, au contraire de ce qui était prévu en 806 , ces deux « centres périphériques » n'étaient pas appelés à fédérer autour d'eux des ensembles plus vastes, les deux rois étant censés rester dans la dépendance de leur aîné, tandis que les deux royaumes ne constitueraient que deux annexes de l'hyper centre impérial.

10 La clause de l'ordination de 817 relative à la réception des ambassades est sur ce point significative: aucune réelle centralité n'est reconnue aux deux royaumes périphériques, puisque la représentation de l'ensemble carolingien vis-à-vis de l'étranger reste l'apanage du seul centre impérial, vers lequel les deux rois devraient 
faire acheminer toute ambassade étrangère touchant leur frontière ${ }^{18}$. L'ordinatio prévoit donc un système dans lequel les périphéries restent sous l'étroite dépendance du centre, dépendance matérialisée par l'absence de pleine autonomie politique des cadets-rois, et par le contrôle exercé par l'aîné-empereur. Ce système restait encore à créer, et il semble avoir trouver une certaine réalité dans les années suivantes : ainsi, au contraire de ce qui se passait sous Charlemagne ${ }^{19}$, on ne trouve pas de traces de négociations menées avec des ambassades par Louis le Germanique ou Pépin entre 817 et 830 . Au contraire, les ambassades bulgares touchant les confins de la Bavière en 824-825 sont acheminées auprès de Louis le Pieux selon une procédure qui rappelle particulièrement celle décrite dans l'ordinatio ${ }^{20}$. Jusqu'en 830 , le rapport centrepériphérie se fait largement à l'avantage du centre, tant du fait de la prégnance du modèle familial qui soumet les rois-fils à l'empereur-père ${ }^{21}$, que de l'organisation d'un réseau de liaisons efficaces entre la zone centrale et les marges. En rupture avec les pratiques de ses prédécesseurs, Louis ne quitte quasiment jamais la zone entre Meuse et Rhin : le centre géographique de l'empire tend à coïncider avec son centre politique et symbolique, c'est-à-dire avec la présence impériale.

11 L'autonomisation des deux cadets induite par les crises successorales des années 830 remettent sérieusement en question ce schéma. La rupture de l'hyper centre fut consacrée par le développement d'une bipolarité entre Rome et Aix, notamment du fait de l'action de Lothaire dans le gouvernement de l'Italie à partir de la négociation de la Constitutio Romana (825) ${ }^{22}$ et, surtout, après sa relégation au-delà des Alpes en 834 . Au temps des navettes du fils fidèle, venu incarner l'autorité impériale en Italie, succède une scission entre le nord des Alpes, aux mains de Louis, et le sud, soumis à Lothaire ${ }^{23}$. Le co-empereur crée un nouveau centre de légitimité autour de lui en Italie, affaiblissant ainsi l'axe Rome-Aix, qui ne forme plus tant un axe central qu'un lien entre deux centres autonomes, séparés par un obstacle naturel de plus en plus perçu comme une frontière: les Alpes ${ }^{24}$. Renouant avec une vieille tradition lombarde, Lothaire fait ainsi fortifier les cluses pour se prémunir au Nord de toute intervention paternelle ${ }^{25}$. L'implosion de l'hyper centre et la confirmation de la marginalisation de l'espace alpin sont entérinées par Lothaire lui-même quand, dès 844 , il fait le choix de confier l'administration du royaume d'Italie à son fils Louis, puis par la consécration de ce partage lors de son retrait du pouvoir en $855^{26}$. Périphérie commune aux trois royaumes des héritiers de Lothaire, les Alpes assument alors pleinement leur statut de voie de communication essentielle.

L'arc alpin, ou qu'une zone frontière n'est pas forcément une zone marginale

Cette position périphérique de l'arc alpin relativement aux centres du pouvoir carolingien est donc avant tout un fait géographique qui n'exclut en rien la possibilité d'une certaine centralité fondée sur sa position d'interface, notamment dans le cadre du développement des communications politiques entre le nord et le sud des Alpes.

Une périphérie en position d'interface

13 Les Alpes centrales avaient déjà joué un rôle majeur dans les communications internes à l'Empire romain puis dans le "grand jeu » diplomatique entre Francs, Burgondes et Ostrogoths au début du $\mathrm{VI}^{\mathrm{e}}$ siècle ${ }^{27}$. Dès le $\mathrm{I}^{\mathrm{er}}$ siècle, des aménagements facilitaient le passage des cols, telle la route carrossable qui permettait le franchissement du Julier et du Septimer par des troupes et des convois de vivre ${ }^{28}$.

14 Ce statut d'interface est réactivé aux $\mathrm{VII}^{\mathrm{e}}$ et $\mathrm{VIII}^{\mathrm{e}}$ siècles par le développement d'une relation privilégiée entre les Francs et les Lombards ${ }^{29}$, puis, à partir des années 750, 
avec la papauté. Deux lieux de passage jouent alors un rôle essentiel, du fait de l'accord par lequel les Lombards avaient cédé Suse et Aoste aux Francs ${ }^{30}$ : il s'agit du Grand Saint-Bernard et du Montcenis. Deux fondations permettent aux Francs de contrôler ces cols et de fournir des étapes facilitant leur franchissement. Saint-Maurice d'Agaune, tout d'abord, sur le versant sud du Valais, avait été fondé par le roi Burgonde Sigismond en 515, dans un double but : asseoir la légitimité de sa dynastie sur le culte des martyrs thébains et tenir le passage des Alpes, qui constituait son principal avantage stratégique vis-à-vis du Goth Théodoric et du Franc Clovis ${ }^{31}$. Ce centre légitimant n'empêche pourtant pas la chute du royaume burgonde et Saint-Maurice s'enfonce alors dans un long silence dont on ne le voit émerger qu'à la fin de 753, quand le pape Étienne II, en route vers la Francie, y fait étape. Le statut de Saint-Maurice est alors problématique, puisque selon le témoignage du rédacteur de la Vita Stephani insérée dans le Liber Pontificalis :

De là, Dieu lui montrant le chemin, il arriva très vite aux cluses des Francs. Et lorsqu'il s'y fut engagé avec ceux qui l'accompagnaient, à l'instant, il chanta les louanges du Dieu tout puissant. Et ayant entrepris la traversée des montagnes, le très bienheureux pontife arriva sain et sauf, grâce à Dieu, avec toute sa suite au vénérable monastère du saint martyr du Christ Maurice, où il avait été convenu que lui et le roi se retrouveraient à égalité. Il y passa quelques jours, et c'est là que mourut le primicier Ambroise d'une mauvaise fièvre. L'abbé Fulrad et le duc Rotard envoyés par le très excellent roi des Francs Pépin arrivèrent alors en ce vénérable monastère ; et ils demandèrent au très saint pontife d'avancer vers leur roi. Et ils le guidèrent avec toute sa suite avec de grands honneurs ${ }^{32}$.

Saint-Maurice est situé au-delà de la frontière entre Francs et Lombards, matérialisée par les clusae Francorum, mais ne semble pourtant pas être placé sous le pouvoir de Pépin, puisque les deux hommes devaient d'abord s'y retrouver pariter, sur un pied d'égalité. L'abbé de Saint-Maurice était l'évêque Wilchaire de Nomentum, personnage au destin complexe. Né en Provence et sans doute apparenté au rector Abbon ${ }^{33}$, il devint archevêque d'Arles et fut l'un des premiers francs à recevoir le palium à Rome à la fin du principat de Charles Martel ${ }^{34}$, dont il avait reçu l'abbatiat de Saint-Maurice ${ }^{35}$. Chassé du siège de Vienne, il se réfugia à Rome où il devint évêque de Nomentum ${ }^{36}$. Il devint ensuite archevêque de Sens et l'un des principaux intermédiaires entre les Francs et Rome ${ }^{37}$.

16 La personnalité de son abbé est donc riche en enseignements sur le statut de cette abbaye en double position de périphérie et d'interface entre l'Italie et le monde franc. C'est le retour de Wilchaire en Francie qui assura son basculement définitif dans l'ensemble carolingien.

17 La nomination de Wilchaire à l'abbatiat d'Agaune coïncide avec un projet global de reprise en mains de l'arc alpin par Charles Martel, en lien avec sa politique d'alliance avec le roi des Lombards Liutprand ${ }^{38}$. C'est dans la même période qu'a lieu le rapprochement entre le rector Abbon, issu d'une puissante famille richement possessionnée dans toutes les Alpes occidentales et le princeps Francorum qui assure le passage du monastère de Novalèse, fondé par Abbon, et donc de la route du Montcenis, sous influence franque ${ }^{39}$. Or, la fondation alpine possédait des biens de part et d'autre de la frontière entre Francs et Lombards ${ }^{40}$. Là encore, donc, on se trouve dans une zone en double situation de périphérie et d'interface. Le centre autonome que voulurent y créer les patrices de Provence et la famille d'Abbon y avorte à la suite de l'intervention franque. 
18 Dans la même période, l'espace alpin central continue à échapper aux Carolingiens. La vallée du Haut Adige est en effet la voie privilégiée des relations entre Bavarois et Lombards, concrétisées par les mariages de Liutprand avec la princesse agilolfide Guntrude et, dans les années 760, de Tassilon avec une fille du roi Didier. Entre le Grand Saint-Bernard et le Vintschgau/Val Venosta subsiste un espace spécifique, totalement alpin, qui contrôle le passage des cols grisons et, tout en liant des relations avec l'ensemble des autres partenaires, développe sa propre centralité autour du siège de Coire ${ }^{41}$. Il s'agit de la Rhétie ${ }^{42}$.

19 Le rôle de la périphérie alpine dans l'émergence d'autonomies périphériques en Bavière, en Provence et surtout en Rhétie amène une question : une périphérie peutelle générer sa propre centralité ? En d'autres termes, un espace en position d'interface entre deux centres (ou plus) peut-il tirer parti de sa position pour se construire en centre alternatif? En suivant les linéaments de l'histoire de l'arc alpin dans le monde carolingien, il apparaît que cette possibilité, ouverte par l'affaiblissement des centres extérieurs, est sans cesse contrebalancée par leur renforcement périodique.

Des autonomies périphériques à la "périphérie centrale "

20 En s'inspirant du schéma d'évolution qu'a posé R. Kaiser pour l'espace rhétique ${ }^{43}$, il est possible de poser les grandes lignes des relations entre les carolingiens et leurs périphéries alpines fondées sur une alternance, de la fin du vII au début du $\mathrm{IX}^{\mathrm{e}}$ siècle, des mouvements d'autonomisation de centres alpins et de réintégration en périphérie du monde franc.

21 À la fin du VII siècle, je l'ai dit, on assiste à l'établissement d'autonomies périphériques : des marges disputées entre Francs, Lombards et Bavarois deviennent le centre de pouvoirs propres aux mains des aristocraties locales, en particulier autour de Coire, tenue par les Zacconides qui confondent les charges d'évêque et de praeses. Le même phénomène se manifeste autour des possessions alpines des Abbonides de Provence. Les testaments de Tellon de Coire ${ }^{44}$, un "faux sincère » $d u$ Ix ${ }^{e}$ siècle, et d'Abbon de Novalèse témoignent de la manière dont ces familles ont appuyé leur pouvoir sur un réseau dense de biens fonciers, de fondations ecclésiastiques, de fidélités et, dans le cas de Coire, de forteresses tenant l'accès aux cols. De façon générale, ces réseaux se densifient dans les vallées qui permettent d'accéder aux passes (voir la carte en annexe).

22 Mais ces deux documents témoignent aussi de la façon dont, dès les années 730 en Provence, dans les années 760 en Rhétie, ces familles mettent à l'abri leurs biens en en faisant don à leurs fondations monastiques afin de les préserver face à l'émergence de la puissance carolingienne. L'histoire du testament de Tellon témoigne de cette pression, puisque le document qui est conservé est en fait une forgerie réalisée autour de 806 à partir de documents épars du siècle précédent et destinée à préserver les droits du monastère de Disentis sur des biens dont il était difficile de déterminer s'ils provenaient des possessions familiales Zacconides ou des «bénéfices » attachés à leurs charges, alors que le nouveau comte, Hunfrid, a reçu pour mission de Charlemagne de mettre fin à la confusion des honneurs publics et ecclésiastiques à Coire ${ }^{45}$.

23 Cette normalisation des structures politiques rhétiques s'intègre dans un processus global de suppression des spécificités de ces périphéries alpines, comme le retour définitif de Saint-Maurice, "monastère pontifical», dans l'orbe carolingienne, ou la protection royale offerte aux fondations familiales zacconides et abbonides. Cela permet aux carolingiens de disposer d'un réseau de monastères royaux qui quadrillent 
l'espace alpin et contrôlent les cols : Novalèse, Saint-Maurice, Disentis. Il faut encore y ajouter le siège de Coire et, en aval, le monastère de Saint-Gall, ainsi que les fondations carolingiennes de Pfäffers, Zurich et Müstair. Ce réseau s'associe à celui des sièges comtaux et ministériaux, des forteresses, des auberges publiques et refuges qui facilitent les communications transalpines en offrant autant d'étapes commodes aux voyageurs (voir la carte en annexe). Un document comme le Polyptyque rhétique de 843, qui détaille les possessions de Pfäffers, décrit ainsi minutieusement les forteresses et les étapes dépendantes du monastère, et les sièges publics sous la juridiction desquels elles sont placées ${ }^{46}$.

Cette "périphérie au centre ", à la fois éloignée des lieux du pouvoir et densément organisée par l'administration carolingienne en raison de son rôle dans les communications stratégiques, conserve donc, malgré la perte de son statut de frontière en 774, un rôle actif d'interface entre le cœur du pouvoir carolingien et le pôle romain au rôle croissant.

Le processus d'éclatement de l'empire lancé à Verdun permit alors, grâce à l'existence de ce réseau dense, de générer de nouveaux centres de pouvoir qui s'appuyèrent sur les points de passages, notamment du fait du rôle stratégique qu'ils prennent avec la séparation progressive de la Lotharingie et de l'Italie entre 844 et 855 , et la disparition du pouvoir lotharingien autonome en 869 .

L'enclenchement de la réaction en chaîne : du divorce de Lothaire à la fondation du royaume de Transjurane

26 L'étude des processus qui accompagnent l'implosion du monde carolingien dans les régions alpines peut s'appuyer sur les études déjà menées sur les cas bavarois ${ }^{47}$ et aquitains ${ }^{48}$. La séparation entre Lotharingie et Italie, puis la compétition entre les princes transalpins pour le contrôle de cette dernière et l'accès à la couronne impériale sont les deux phénomènes fondamentaux qui président à cette évolution. En outre, plus précocement peut-être qu'ailleurs, on voit de grandes familles, implantées localement dans la Péninsule et alliées aux Carolingiens, intervenir dans cette compétition pour la maitrise d'un espace d'interface essentiel. De ces rivalités naissent de nouvelles centralités.

Les Alpes, objet de compétition stratégique

entre les Carolingiens et les grandes familles

Dès les premières années du règne de Louis II d'Italie, la réactivation du rôle frontalier des Alpes par la tripartition du royaume de Lothaire entraîne un intérêt nouveau pour cet espace. Le royaume provençal de Charles dont relève les cols occidentaux, est mal accepté par ses frères ${ }^{49}$. La mort précoce de Charles de Provence entraîne ainsi une négociation tendue entre Louis et Lothaire II, à l'issue de laquelle un accord est trouvé qui transfère à Louis les diocèses de Genève, Lausanne et Sion. Cependant, les voies d'accès alpines occidentales ${ }^{50}$, et en particulier le pôle essentiel de Saint-Maurice d'Agaune, dont l'abbatiat laïc avait été confié par Lothaire à son beau-frère Hubert ${ }^{51}$, restent dans le royaume du Nord. Les possessions du monastère sont mieux connues dans cette période, grâce à l'édition partielle de son chartrier ${ }^{52}$. Elles se sont développées, au détriment de Novalèse, dans la plus grande partie des Alpes occidentales et débordent largement dans le Val d'Aoste, sur le versant sud. Un espace est plus particulièrement densément organisé par le monastère : il s'agit de la voie d'accès qui, de Genève par le Chablais et le Valais mène au pied du Saint-Bernard par la haute vallée du Rhône. Dans cette zone, l'action du monastère est très semblable à ce 
qui se retrouve autour des centres rhétiques de Coire, Disentis et Pfäffers. On observe ces zones d'influence d'Agaune et de Coire dans les parties figurées en grisé sur la carte en annexe.

Un autre document, daté de 855 , témoigne de ce regain d'intérêt à l'autre extrémité des Alpes centrales : il s'agit d'un diplôme de Louis le Germanique ${ }^{53}$ par lequel, en présence des représentants de Louis d'Italie, il tranche un désaccord entre les sièges de Freising et de Trente au sujet d'un bien dans les environs de Bozen/Bolzano, dans le Vintschgau/Val Venosta, jadis disputé entre Bavarois et Lombards et dont le statut n'avait semble-t-il pas été clairement tranché après le rattachement de ces deux ensembles au regnum Francorum ${ }^{54}$. Le souverain transalpin, qui possède déjà la Rhétie et l'Engadine, confirme sa position en obtenant la reconnaissance de son pouvoir sur la zone concernée, par le biais le siège de Freising, et par conséquent le contrôle d'un voie de passage qui constitue le débouché naturel de ses possessions sur le versant nord ${ }^{55}$.

À partir des années 850 , toutes les grandes voies de communication alpines sont aux mains des souverains du nord, ce qui va jouer sur l'avenir des relations entre les deux versants du massif. La mort de Lothaire II en 869 voit le passage des cols occidentaux sous la puissance de Charles le Chauve. En 875 puis en 877, en route pour l'Italie, il emprunte le Grand Saint-Bernard et fait étape à Saint-Maurice, dont l'abbatiat laïc est passé à Boson, lui aussi son beau-frère, et cousin de Hubert ${ }^{56}$.

Les Bosonides, groupe familial auquel appartiennent ces deux personnages, possessionnés aussi bien en Lotharingie qu'en Italie du Nord, font de ce centre de SaintMaurice une base de leur pouvoir, en utilisant l'affaire du divorce de Lothaire II dans laquelle ils sont impliqués à double titre ${ }^{57}$. Entre 859 et 864 , Hubert entre en révolte ouverte contre Lothaire et fonde un embryon de principauté alpine autour de son monastère ${ }^{58}$, désorganisant les relations transalpines pourtant vitales pour le roi engagé dans une controverse ardue avec Rome.

31 Après sa mort et la disparition du royaume de Lothaire, c'est son neveu Boson qui, par la faveur de Charles, hérite de cet ensemble, dont il fait l'un des pôles de son royaume de Vienne à partir de 879. Une rencontre entre Charles le Gros et les rois Louis III et Carloman à propos de l'action à mener contre l'usurpateur Boson en 881, alors que Charles s'apprête à gagner Rome pour y recevoir la couronne impériale, se tient d'ailleurs significativement à Orbe ${ }^{59}$, au nord-ouest de Genève, et prélude à une offensive par laquelle les carolingiens libèrent cette voie stratégique. Empereur, Charles place l'un de ses principaux fidèles, Liutward, sur le siège épiscopal de Verceil, qui contrôle le débouché sur le versant sud de la route du Saint-Bernard ${ }^{60}$. Dans les années suivantes, il privilégie néanmoins, pour des raisons tant stratégiques que géographiques, les passes grisonnes, entrant en Italie par Trente, qui avait déjà été le lieu de rencontre privilégié de Louis le Germanique et de Louis d'Italie puis de sa veuve Engelberge ${ }^{61}$. Cet itinéraire était en effet le plus court depuis la Germanie, zone de résidence principale de Charles, et restait mieux tenu que les cols occidentaux en proie à la compétition entre les aristocrates.

Nouveaux centres et réactivation des réseaux

Le royaume de Vienne est vite affaibli par l'intervention militaire carolingienne, par ses ambitions italiennes, mais aussi par l'intervention d'une autre famille dans sa zone d'action : les Welfs ${ }^{62}$. Après 843 , la famille s'était scindée en deux rameaux qui avaient construit leur influence parallèlement en Bourgogne et en Thurgovie. La branche occidentale, proche de Charles le Chauve, conclut une alliance matrimoniale avec les 
Bosonides, eux aussi membres de cette Königsnähe. Mais c'est également un Welf, Conrad, qui fut à l'origine de la chute d'Hubert en 864 et hérita de ses honores alpins ${ }^{63}$. Ils investirent ainsi l'espace qui constituait la marge entre leurs possessions bourguignonnes et jurassiennes : la zone alpine. En 888, le Welf Rodolphe, dont la sœur Adélaïde avait épousé le frère de Boson, est proclamé roi en Bourgogne transjurane par l'aristocratie réunie à Saint-Maurice d'Agaune où il est couronné ${ }^{64}$. Saint-Maurice devient le pôle majeur de cette dynastie ${ }^{65}$, ce qui lui permet de s'affirmer face au dernier Carolingien " légitime », puisque Arnould de Carinthie doit durement batailler contre Rodolphe et, finalement, reconnaître son pouvoir pour franchir le Saint-Bernard à son retour d'Italie en $894^{66}$. Saint-Maurice assure aussi la filiation fictive entre le royaume burgonde $d u v^{e}$ siècle et le royaume de Transjurane $d u x^{e}$ siècle, à travers la réactivation des légendes de fondation royale burgonde et le culte de la Sainte Lance qui y était conservée ${ }^{67}$. Saint-Maurice, au-delà de sa fonction d'interface essentiel entre le nord et le sud des Alpes, devient donc un centre de légitimité appelé à se perpétuer au-delà de l'affaiblissement des Rodolphiens, par la captation de la précieuse relique et du culte de saint Maurice par les Ottoniens, puis par la translation des reliques mêmes du saint dans le royaume capétien, où Saint-Louis les fait déposer à Senlis, lieu de l'origine symbolique de sa dynastie. Le centre né à la périphérie ne survit donc pas au retour en force des centres extérieurs, et la victoire de ces derniers se traduit par le déplacement vers le Centre des signes de légitimité générés par la périphérie.

Ce centre transjuran s'est donc fondé sur l'alliance entre les Welfs et les Bosonides. Il se maintient par l'apparition d'une autre relation avec une grande famille alpine: les Hunfridides de Rhétie ${ }^{68}$. Plus tardivement que dans les Alpes occidentales, cette famille a également développé un pouvoir autonome fondé sur le centre de Coire et la réactivation du maillage de l'espace alpin construit depuis l'époque des Zacconides. Ce centre reste néanmoins inachevé, puisque le pouvoir qu'il génère reste subordonné à celui du roi puis de l'empereur de Germanie. La famille hunfridide accède en effet à la tête du duché de Souabe. De plus, cette réussite dynastique se traduit par une marginalisation de l'espace originel au profit des centres traditionnels de l'espace alaman, dont sont originaires les Hunfridides. Plus que Coire, c'est Saint-Gall qui devient leur principal point d'appui. Néanmoins, dans cette expérience d'une principauté rhétique au sein de l'ensemble germanique se décèlent les premiers linéaments d'une identité helvétique.

$\mathrm{Du} \mathrm{VII}^{\mathrm{e}}$ au $\mathrm{x}^{\mathrm{e}}$ siècle, l'histoire alpine est dominée par une alternance d'intégrations en marge d'ensembles plus importants et d'apparitions d'autonomies périphériques. Mais cet espace reste toujours subordonné à un centre, ou plutôt, à des centres extérieurs, ce qui le place en position d'interface entre plusieurs réseaux de pouvoirs. Les affaiblissements périodiques de ces centres se traduisent par une désorganisation de ces réseaux dans les périphéries, qui compromettent leurs communications. Ces « trous structuraux " les empêchent d'accomplir eux-mêmes ce travail d'information et de relations avec l'extérieur, et en particulier avec le centre essentiel que devient Rome dans la période. Des intermédiaires issus des grandes familles aristocratiques franques implantées localement peuvent alors tirer profit de ces interruptions pour réactiver ces réseaux à leur profit et construire leur pouvoir sur cette position d'interface. Mais ces centres périphériques continuent à souffrir de leur éloignement des centres de légitimité traditionnels, ce qui les condamnent à redevenir de simples étapes en marge, même s'ils conservent un rôle stratégique, une fois ces pouvoirs englobants rétablis. 
Ce processus témoigne de la structuration complexe, moléculaire, des réseaux qui à partir d'un "hyper centre", se construisent sur une succession de centres locaux, reliés ou non entre eux, de taille et d'importance variables, qui génèrent eux-mêmes des maillages dans leur voisinage. Au contact entre deux " hyper réseaux », ces centres locaux jouent un rôle d'interface essentiel, surtout lorsqu'ils parviennent à piéger les hyper centres dans des trous structuraux par leurs relations croisées.

Cette analyse permet de comprendre la façon dont les Alpes se sont structurées en une frontière perméable, cadre d'une société spécifique à l'identité problématique, qu'organise des centres liés à l'extérieur, mais dont le rôle d'interface permet l'autonomisation.

\section{NOTES}

1. Le sujet est vaste et a fait l'objet d'études abondantes. Dans un souci de clarté, je me bornerai à citer, dans cette note et celles qui suivent, les études de cas qui m'ont servi de modèle : C. LAURANSON-RosAz, L'Auvergne et ses marges (Velay, Gévaudan) au VIII et IX siècle. La fin du monde antique?, Le Puy-en-Velay, 1987.

2. N. BROUSSEAU, Étude comparée des diplômes de Louis le Germanique et de Charles le Chauve (829-877), DEA de l'Université Paris I et Thèse de l'École Nationale des Chartes, 2002.

3. G. BÜHRER-THIERRY, Évêque et pouvoir dans le royaume de Germanie (876-973), Paris, 1997 ; В. BigotT, Ludwig der Deutsche und die Reichskirche im Ostfränkischen Reich (826-876), Husum, 2002.

4. R. BRUNET, Les Mots de la Géographie, art. «Centre », Paris, Reclus (Dynamique du Territoire), 1992, p. 88.

5. Ibid., "Centre et Périphérie », p. 89.

6. Convention adoptée par les géographes, v. R. BRUNET, op. cit., p. 89.

7. R. S. BURT, « Le Capital Social, les trous Structuraux et l'entrepreneur ", Revue Française de Sociologie, vol. 36, 1995, p. 599-628.

8. R. BRUNET, Les mots de la Géographie, op. cit., art. «Interface », p. 258.

9. Dans un diplôme délimitant le nouveau diocèse de Constance donné entre 632 et 639, Dagobert $\mathrm{I}^{\text {er }}$ définit ainsi les frontières nord du diocèse de Coire comme la vraie frontière du royaume, la partie alpine située au sud restant une marche entre ses possessions et le royaume lombard : Bündner Urkundenbuch, E. MEYER-MARTHALER et F. PERRET éd., Coire, 1955-2001, n 8, p. 7 (désormais abrégé : Bund. Urk.). Ce texte, inséré dans un diplôme de Frédéric $\mathrm{I}^{\mathrm{er}}$ pour Constance du 2 novembre 1155 (Thurgauer Urkundenbuch, II, p. 42), a longtemps été considéré comme un faux. R. KAISER (Churrätien im frühen Mittelalter, Bâle, 1998) a montré qu'il pouvait être considéré comme un témoin d'une délimitation qui eut réellement lieu sous Dagobert après l'érection du siège de Constance et le règlement définitif des querelles frontalières entre Francs et Lombards dans les Alpes.

10. Ainsi, Frédégaire ne parle que rarement des Alpes, mais plutôt de l'espace «transjuran » (partes transiuranae), par exemple, Chronique, O. DevilLERs et J. MEYERS éd., 
Turnhout, 2001, col. 43 (613-617), p. 124. : « De internicione Erpone ducis. Cum anno 30. regni sui in Burgundia et Auster regnum arepuisset, Herpone duci genere Franco locum Eudilanae in pago Ultraiorano instituit. »

11. MGH, Epistolae Karolini Ævi II, $\mathrm{n}^{\circ} 77$, p. 102 : «Dulcissimo patri Remigio episcopo Albinus humilis filius salutem. Hunc nostrum negociatorem Italiae mercimonia ferentem, his litteris tuae paternitatis commendo protectionis, ut per vias vestrae patriae tutus eat et redeat ; et in montium claustris a vestris non teneatus tolneariis constrictus, sed per latitudinem caritatis latam habeat eundi et redeundi semitam. Iubeas, obsecro, orare pro anima Ioseppi discipuli mei. "Floreas et proficias de virtute in virtutem, donec videas laetus et gaudens Deum tuum in Sion." Memorque mei sis, sansctissime pater, obsecro. »

12. P. G. GEARY, Aristocraty in Provence. The Rhône basin at the dawn of the carolingian age, Stuttgart, 1985.

13. Frédégaire, Chronique, IV, 45, p. 126.

14. H. BÜTTNER, « Die Bündner Alpenpässe im frühen Mittelalter », dans H. AUBIN, E. ENNEN, H. KELLENBENZ dir., Beiträge zur Wirtschafts- und Stadtgeschichte, Wiesbaden, 1965, p. 242-252.

15. Karoli Divisio Regnorum, MGH, Capitularia Regum Francorum I, p. 126-130, et plus particulièrement col. 1 et 2, p. 126.

16. Ordinatio Imperii, MGH, Capit. I, $\mathrm{n}^{\circ} 136$, p. 270-273, et plus particulièrement col. 2 et 8 , p. 270 et 271.

17. Divisio regnorum, cap. 2, 3 et 4, p. 126.

18. Ordinatio Imperii, cap. 8, p. 271.

19. Par exemple, les négociations menées par Louis le Pieux avec les musulmans d'Espagne et les Asturiens : Anonymi Vita Hludowici Imperatoris, chap. 5, 8, p. 609 et 611.

20. MGH, Scriptores I, Annales Regni Francorum, p. 212 : «Quo cum venisset et ibi natalem Domini celebrasset, allatum est ei, quod legati regis Bulgarorum essent in Baioaria ; quibus obviam mittens ipsos quidem usque ad tempus congruum ibidem fecit operiri. » 21. Les dernières lignes du prologue de l'Ordinatio, p. 271, sont très claires sur la soumission que les fils doivent conserver « comme il convient » à l'égard d'un père.

22. Ann. Reg. Franc., p. 160-163 [passim], p. 164-166 [passim] et p. 167-168.

23. Ainsi, en 838, Louis et Lothaire se rencontrent dans les Alpes qui marquent la limite de leurs zones respectives : Annales Bertiniani, F. Grat éd., Paris, 1964, p. 9 : «Post haec peractis sacrae nativitatis, apparitionis atque oblationis dominicae sollempnitatibus, inchoatisque quadragesimae ieiuniis, imperatori sermo innotuit, Hlodowicum fratris Hlotharii intra Alpium septa colloquium expetisse. »

24. Le Preceptum mercatorum de 828 est ainsi le premier document franc qui définit clairement les cluses alpines comme lieu de prélèvement douanier : $M G H$, Formulae imperiales, $\mathrm{n}^{\circ} 37$, p. 314.

25. Ann. Bert., p. 8 : « Hlotharius autem clusas in Alpibus muris firmissimis arceri praecepit. "

26. Ann. Bert., p. 45 : «Dispositoque inter filios, qui secum morabantur, regno, ita ut Lotharius cognomen eius Franciam, Karlus vero Provinciam optinerent, intra sex dies vita decessit quarto Kalendarum Octobrium, [...]. » Lothaire avait confié à son fils Louis le gouvernement de l'Italie dès 846 et l'avait fait couronné empereur à Rome dès 850 : Ann. Bert., p. 37.

27. R. KAISER, Churrätien, op. cit., cf. n. 9, p. 14-18. 
28. I. H. RiNGEL, « Kontinuität und Wandel : die Bündner Pässe Julier und Septimer von der Antike bis ins Mittelalter » dans F. BURGARD et A. HAVERKAMP dir., Auf den Römerstrassen ins Mittelalter, Mayence, 1997, p. 211-296.

29. Notamment la conclusion d'une amicitia perpetua en 617 : Frédégaire, Chronique, chap. 45, p. 126.

30. Ibid.

31. R. KAISER, art. « Les Burgondes », Dictionnaire historique de la Suisse, Berne, 2003-2005, http://www.dhs.ch/externe/protect/textes/f/F8028.html.

32. Vita Stephani II. insérée dans le Liber Pontificalis, L. DuchESNE, éd., Paris-Rome, 1955, t. I, col. XIX, p. 445 : « Deo praevio, ad Francorum coniunxit clusas. Quas ingressus cum his qui cum ei reant, confestim laudes omnipotenti Deo reddidit ; et coeptum gradiens iter, ad venerabile monasterium sancti Christi martyris Mauricii, in quo et constitutus erat pariter se cum Francorum rege conveniri, annuente domino, sospes hisdem beatissimus pontifex cum omnibus qui cum eo erant advenit. In quo et aliquantis demorantes diebus, [et ibi Ambrosius primicerius febre correptus defunctus est] coniunxerunt in praedicto venerabili monasterio Fulradus abbas et Rotardus dux directi a sepefato Pipino excellentissimo Francorum rege petentes eundem sanctissimum pontificem ad suum progredi regem. Quem et cum magno honore cum omnibus qui cum eo erant ad eum deduxerunt. »

33. P. GEARY, Aristocraty in Provence, op. cit., cf. n. 12.

34. Liber Pontificalis, I, p. 421.

35. Chronique d'Adon de Vienne, PL, CXXII, p. 121-123.

36. Liber Pontificalis, n. 25 et 26, p. 457.

37. K. RIESENBERGER, Prosopographie der Papstslicher Legaten, p. 12.

38. J. JARNUT, « Die Adoption Pippins durch König Liutprand und die Italien Politik Karl Martells ", dans J. JARNUT, U. NONN et M. RICHTER dir., Karl Martell in seiner Zeit, actes du colloque de Bad-Homburg, 26-28 février 1992, Sigmaringen, 1994, p. 217-227.

39. P. GEARY, Aristocraty in Provence, op. cit., cf. n. 12.

40. Testament d'Abbon, dans P. GEARY, ibid., p. 38-78, en particulier col. 8 et 9, p. 39.

41. Deux épitaphes conservées à Coire gardent la trace de contacts avec le Vintschgau bavarois et le Trentin (Bündner Urkundenbuch, $\mathrm{n}^{\circ} 11$, p. 8 et $\mathrm{n}^{\circ}$ 12, p. 9). La Rhétie appartenait nommément au royaume des Francs depuis les années 530 (R. Kaiser, Churrätien). Ses cols étaient en outre essentiels aux relations entre les Bavarois et les Lombards, et même, à la liaison entre le cœur de l'espace bavarois et le Vintschgau, espace en lui-même disputé entre Bavarois, Rhètes et Lombards. On manque malheureusement cruellement de sources sur ce sujet.

42. R. KAISER, « Autonomie, Integration, bilateraler Vertrag : Rätien und das Frankenreich im frühen Mittelalter », Francia, 29/1, 2002, p. 1-27.

43. Ibid., p. 3.

44. Bünd. Urk., $\mathrm{n}^{\circ} 17, \mathrm{p} .13-23$, cette source, dont l'authenticité a été attaquée et dans laquelle certains ont voulu voir une forgerie postérieure a été réhabilitée par R. Kaiser, qui a montré qu'elle reposait sur un original aujourd'hui perdu du VIII ${ }^{\mathrm{e}}$ siècle, $\mathrm{v}$.

R. KAISER, Churrätien, p. 36.

45. R. KAISER, Churrätien, p. 36.

46. Polyptyque Rhétique, dans Bünd. Urk., I, annexe, p. 375-396.

47. J. JAHN, Ducatus Baiuwariorum. Das bairische Herzogtum der Agilolfinger, Stuttgart, 1991. 
48. A. LAURAnçON-RoSAZ, L'Aquitaine Carolingienne, op. cit., n. 1.

49. Ann. Bert., p. 47 : « Ludoicus imperator Italiae et Hlotharius, frater eius, rex Franciae, cum Karlo puero, germano suo, apud Urbam conveniunt. Ubi adeo pro regni paterni portionibus dissident, ut pene armis inter sese decernant ; Karlo tamen, fratri suo, Provinciam et ducatum Lugdunensem iuxta paternam dispositionem distribuunt, eripientibus eum a fratre Lothario obtimatibus, qui illum moliebatur in clericum tonsurare. » Cet accord fait suite à l'invasion d'une partie du royaume de Charles par Louis, qui revendiquait les frontières de l'Italie de Pépin, l'année précédente, ibid., p. 46. 50. Ann. Bert., p. 53 : « Lotharius fratri suo Ludoico Italorum regi quandam regni sui portionem adtribuit, ea videlicet quae ultra Iuram montem habebat, id est Genuvam, Lausonnam et Sedunum civitates, cum episcopatibus, monasteriis et comitatibus, praeter hospitale quod est in monte Iovis et Pipincensem comitatum. »

51. RÉGINON, Chronique, MGH, Script. in us., p. 570 : « Anno dominicae incarnationis DCCCLVIIII. Lotharius Hucberto abbati ducatum inter Iurum et montem Iovis commisit, eo quod tunc fidelissimus putaretur, utpote affinitate coniunctus propter sororem Thietbirgam. »

52. R. BECCI, Le Chartrier de l'Abbaye de Saint-Maurice d'Agaune (1128-1292) : édition et présentation, 5 vol., thèse pour le diplôme d'archiviste paléographe, École nationale des Chartes, Paris, 1997, un nombre croissant de documents sont consultables en édition électronique sur le site de la Fondation des Archives Historiques de Saint-Maurice : http://www.aasm.ch/fondation.html.

53. MGH, Dip. I (DLG), $\mathrm{n}^{\circ} 72$, p. 101-102.

54. J. JARNUT, « Bozen zwischen Langobarden, Bayern und Franken », dans Bozen, von den Anfängen bis zur Schleifung der Stadtmauern, Bolsano/Bozen, 1991, p. 135-141. 55. C'est d'ailleurs à Trente, au débouché de cette voie, que les deux Louis se rencontrent deux ans plus tard pour régler définitivement la querelle entre les sièges de Freising et Trente : DLG 85, p. 122.

56. Ann. Bert., p. 127 : « Kalendis Septembribus iter suum incoepit, et per Sancti Mauritii monasterium pergens, montem Iovis transiit et Italiam ingressus fuit. » En 877 (p. 136), Charles s'arrête à Orbe, puis gagne Verceil où il est accueilli par Jean VIII.

57. F. BOUGARD, « En marge du divorce de Lothaire II : Boson de Vienne, le cocu qui fut fait roi ?", Francia, n 27/1, 2001, p. 33-51.

58. RÉGINON, Chronique, p. 577 : « Hac tempestate Hucbertus abba, frater Thietbirgae reginae, contra Lotharium regem rebellare exorsus est ; collecta quippe predonum valida manu rapinas coepit exercere interfectisque aut fugatis Lotharii fidelibus, qui finitima possidebant loca, agros villasque eorum suae factionis sectatoribus distribuit. Ad huiuscemodi temeritatem comprimendam Lotharius rex semel, iterum atque tertio exercitum produxit, frequenter etiam cum ducibus armatorum multitudinem direxit ; sed minime hanc presumptionem extinguere potuit, quia loca inaccessibilia inter Iurum et Alpes Penninas seditiosis munitissimum prebebant receptaculum et regi exercituique eius propter concava vallium et prerupta montium artissima itinera difficilem ingressum. [...] Attamen idem acephala Hucbertus novissime a Conrado comite peremptus est iuxta castrum, quod Urba dicitur. »

59. Ann. Bert., p. 232.

60. G. BÜHRER-THIERRY, Évêques et Pouvoir dans le Royaume de Germanie, cf. n. 3.

61. DLG, $\mathrm{n}^{\circ} 85$, p. 122, et Ann. Bert., p. 182-183 (872). La même année, Engelberge propose à Charles le Chauve une rencontre à Saint-Maurice d'Agaune. 
62. R. LE JAN, Famille et pouvoir dans le monde franc ( $\mathrm{VII}^{\mathrm{e}}-\mathrm{X}^{\mathrm{e}}$ siècles). Essai d'anthropologie sociale, Paris, 1995.

63. RÉGINON, p. 577.

64. RÉGINON, p. 598 : «Per idem tempus Ruodolfus filius Cuonradi, nepos Hugonis abbatis, de quo supra meminimus, provintiam inter Iurum et Alpes Penninas occupat et apud sanctum Mauritium adscitis secum quibusdam primoribus et nonnullis sacerdotibus coronam sibi imposuit regemque se appellari iussit. »

65. M. ZUFFEREY, Die Abtei Saint-Maurice d'Agaune im Hochmittelalter (830-1258), Göttingen, 1988.

66. RÉGINON, Chronique, p. 606-607 : «Anno dominicae incarnationis DCCCXCIIII. Arnulfus [...] inde conversus per Alpes Penninas Galliam intravit et ad sanctum Mauricium venit. Ruodulfum, quem quaerebat, nocere non potuit, quia montana conscendens in tutissimis locis se absconderat. regionem inter Iurum et montem Iovis exercitus graviter adtrivit. "

67. R. KAISER, loc. cit.

68. M. BORGOLTE, Von Hunfrid bis Burkard, Stuttgart, 1986, plus particulièrement $p$. 181-209. Le roi Rodolphe II de Bourgogne épousa Berthe, la sœur du marquis Bouchard de Rhétie, duc de Souabe au début du $\mathrm{x}^{\mathrm{e}}$ siècle.

\section{RÉSUMÉS}

Le problème de la réorganisation de l'ensemble politique carolingien après le traité de $843 \mathrm{a}$ pu être étudié sous divers angles mettant en valeur ou bien la manière dont chaque royaume se structure ou bien la façon dont, sur les marges, naissent de nouvelles entités organisées autour d'identités culturelles antérieures à la conquête franque et historiquement inscrite dans ces espaces. Ces principautés périphériques sont le fruit d'une dialectique entre les anciens centres du pouvoir franc, entre Loire et Rhin, et les marges. Il s'agit ici d'étudier ce rapport et son évolution à travers un cas limite : l'arc alpin central et occidental, qui tout en étant au centre géographique de l'Europe carolingienne, et sur une voie de passage essentielle reliant le centre carolingien à Rome, se trouve en position de périphérie politique entre les royaumes nés du partage de 843 et de ceux qui suivent. Il s'agira donc de comprendre comment les aristocraties ont pu exploiter cette position spécifique pour y développer des réseaux, bases de la construction de pouvoirs autonomes à la fin du IX ${ }^{\mathrm{e}}$ siècle.

The Nuclear Fission: Ancient and New Centers in the Alpine Region. from the End of the 7th Century to the Beginning of the 10th Century. The new structure of the Carolingian kingdoms born from the Treaty of Verdun (843) was studied under various aspects, including the way they manage to create their own inner structures and the birth, on the borders, of new entities organised by cultural identities who precede the Frankish come over and were historically inscribed in these spaces. These peripheral principalities resulted from a dialectic process between the old centres of the Frankish power, between Loire and Rhine, and the borders. I intend to study this relationship and its evolution through a «border case » : the central and occidental Alps, which were situated geographically at the centre of Carolingian Europe, and on an essential way going from the Carolingian centre to Rome, but were indeed a politic border between the kingdoms resulting 
from the division of 843 and the following. I aim to understand how aristocracies exploited this particular situation in order to build nets that lead to autonomous powers at the end of the Ixth century.

INDEX

Mots-clés : Carolingiens, centre/périphéries, Alpes, réseaux, relations internationales Keywords : Carolingians, center/border, Alps, networks, international relationships

\section{AUTEUR}

RODOLPHE DREILLARD

Université Lille III-Charles-de-Gaulle, UMR 8529 IRHiS, BP 60149, 59653 Villeneuved'Ascq Cedex 